\title{
Spatially resolved detection of crystallized water ice in a T Tauri object ${ }^{\star} \star \star$
}

\author{
A. A. Schegerer ${ }^{1,2}$ and S. Wolf ${ }^{3}$ \\ 1 Helmholtz Zentrum München, German Research Center for Environmental Health, Ingolstädter Landstraße 1, \\ 85758 Neuherberg, Germany \\ e-mail: schegerer@mpia-hd.mpg.de \\ 2 Max-Planck-Institut für Astronomie, Königstuhl 17, 69117 Heidelberg, Germany \\ 3 Universität Kiel, Institut für Theoretische Physik und Astrophysik, Leibnizstraße 15, 24098 Kiel, Germany
}

Received 15 February 2009 / Accepted 29 April 2010

\section{ABSTRACT}

\begin{abstract}
Aims. We search for frozen water and its processing around young stellar objects (YSOs of class I/II). We try to detect potential, regional differences in water ice evolution within YSOs, which is relevant to understanding the chemical structure of the progenitors of protoplanetary systems and the evolution of solid materials. Water plays an important role as a reaction bed for rich chemistry and is an indispensable requirement for life as known on Earth.

Methods. We present our analysis of NAOS-CONICA/VLT spectroscopy of water ice at $3 \mu \mathrm{m}$ for the T Tauri star YLW $16 \mathrm{~A}$ in the $\rho$ Ophiuchi molecular cloud. We obtained spectra for different regions of the circumstellar environment. The observed absorption profiles are deconvolved with the mass extinction profiles of amorphous and crystallized ice measured in laboratory. We take into account both absorption and scattering by ice grains.

Results. Water ice in YLW16A is detected with optical depths of between $\tau=1.8$ and $\tau=2.5$. The profiles that are measured can be fitted predominantly by the extinction profiles of small grains $(0.1 \mu \mathrm{m}-0.3 \mu \mathrm{m})$ with a small contribution from large grains $(<10 \%)$. However, an unambiguous trace of grain growth cannot be found. We detected crystallized water ice spectra that have their origin in different regions of the circumstellar environment of the T Tauri star YLW $16 \mathrm{~A}$. The crystallinity increases in the upper layers of the circumstellar disk, while only amorphous grains exist in the bipolar envelope. As in studies of silicate grains in T Tauri objects, the higher crystallinity in the upper layers of the outer disk regions implies that water ice crystallizes and remains crystallized close to the disk atmosphere where water ice is shielded against hard irradiation.
\end{abstract}

Key words. infrared: stars - accretion, accretion disks - astrobiology

\section{Introduction}

The importance of water (ice) to protoplanetary systems is justified by the following properties: liquid water is a proper solvent for many minerals and organic molecules and allows the formation of complex organic molecules. Water is the reaction bed for the photochemical synthesis of both aromatic compounds (Bernstein et al. 2002) and amino acids (Munoz Caro et al. 2002), even in its solid state. Therefore, rich deposits of water (ice) in protoplanetary disks are assumed to be a requirement for the formation of life as known on Earth. The importance of water ice is in addition emphasized by the assumption that ice planets, such as Neptune, form outside the snowline where water condenses on dust grains. The mass fraction of solid matter in protoplanetary disks abruptly increases at the snowline at least by one order of magnitude (Stevenson \& Lunine1988).

The water ice band at $3.08 \mu \mathrm{m}$, which is caused by a vibrational excitation of the $\mathrm{OH}$ bond in the water molecule, was primarily discovered in the envelopes of numerous deeply embedded protostars (Gillett \& Forrest 1973; Gillett et al. 1975;

* Based on observations collected at the European Organisation for Astronomical Research in the Southern Hemisphere, Chile (proposal 077.C-0794(A)).

$\star \star$ Appendix is only available in electronic form at http://www . aanda.org
Merrill et al. 1976). Frozen water that can be found in dust particles is the most frequent ice molecule in YSOs (Ehrenfreund et al. 1996) ${ }^{1}$. The huge water ice deposit results from its molecular property: in contrast to nonpolar, volatile ice molecules, such as $\mathrm{CO}, \mathrm{CO}_{2}, \mathrm{~N}_{2}$, and $\mathrm{O}_{2}$ ice, $\mathrm{H}_{2} \mathrm{O}$ ice has a high sublimation temperature of $\sim 120 \mathrm{~K}$ depending on the ambient pressure (Davis 2007). The polarity of the water molecule is responsible for its stable adsorption by condensation seeds such as silicate dust grains. Cosmic irradiation and chemical reactions could force the evaporation of water ice (Léger et al. 1985; Hartquist $\&$ Wolf 1990). However, these reactions are impeded by regions with strong shielding and low temperatures (Tielens \& Hagen 1982). This is why the water ice band is commonly observed in the spectra of deeply embedded protostars (Boogert et al. 2004). However, it is assumed that larger deposits of water ice exist in the optically thick regions of circumstellar disks (Fig. 1 in Chiang et al. 2001). This assumption may be supported by the detection of the water ice band at $46.5 \mu \mathrm{m}$ (Creech-Eakman et al. 2002) and $3.08 \mu \mathrm{m}$ (Leinert et al. 2001; Terada et al. 2007) in the spectra of several T Tauri objects.

\footnotetext{
${ }^{1}$ Fourteen different ice compounds, such as $\mathrm{CO}, \mathrm{CO}_{2}$, or $\mathrm{CH}_{4}$ ice are known. Ice molecules are weakly bounded just by van-der-Waals forces or by hydrogen bonds. A chemical compound that can be found in metals, minerals, and diamonds does not form.
} 
The water ice band at $3.08 \mu \mathrm{m}$ in $L$ band $^{2}$ is assumed to be an excellent indicator of the evolutionary status of circumstellar disks (van de Bult 1985). In a protostellar molecular cloud, water ice has a primarily amorphous structure, as many previous studies have shown (e.g., Thi et al. 2006; Pontoppidan et al. 2005). As soon as the temperature exceeds a level of $\sim 80 \mathrm{~K}$, water ice begins to crystallize (Hagen et al. 1981a). This conversion can be detected by monitoring the profile of the optical depth, i.e., the logarithm of the absorption band: as the crystallinity increases, the profile narrows and its maximum shifts to longer wavelengths (e.g., Smith et al. 1989). But the crystalline structure can also be destroyed, e.g., by hard irradiation (Kouchi $\&$ Kuroda 1990). In addition to crystallization, grain growth of ice particles can be inferred from a modification of the optical depth profile. An increase in the dust grain radius results in a higher scattering efficiency, a broadening of the absorption band at long wavelengths, and a shift of the maximum to longer wavelengths. These changes are comparable to those related to the prominent $10 \mu \mathrm{m}$ silicate band (e.g., Bouwman et al. 2001), but the origin of the spectral bands is different. While the emission band of silicate has its origin in the warm disk atmosphere, the absorption band of water ice originates in more embedded, cold regions closer to the disk midplane and far from the central star.

The water ice band interferes with absorption bands of additional materials that are dissolved in water ice or form a complex compound with the water molecule: ammonia hydrate $\mathrm{H}_{2} \mathrm{O} . \mathrm{NH}_{3}$ (minimum at $3.47 \mu \mathrm{m}$ : Merrill et al. 1976; Mukai et al. 1986; Dartois \& d'Hendecourt 2001), ammonia $\mathrm{NH}_{3}$ (2.96 $\mu \mathrm{m}$ : d'Hendecourt et al. 1985; Dartois \& d'Hendecourt 1985), methanol $\mathrm{CH}_{3} \mathrm{OH}$ (3.54 $\mu \mathrm{m}$ : Dartois et al. 2001), hydrocarbons with the vibrational excitations of the $\mathrm{CH}, \mathrm{CH}_{2}$, and $\mathrm{CH}_{3}$ bond $(3.38 \mu \mathrm{m}, 3.42 \mu \mathrm{m}$, and $3.48 \mu \mathrm{m}$ : Duley \& Williams 1984; Chiar et al. 2002), and other chemical compounds (Ehrenfreund et al. 1996). The absorption bands of these molecules are narrow and interfere predominantly with the water ice band at longer wavelengths around $3.6 \mu \mathrm{m}$. They can therefore be distinguished from the water ice band. To study the chemical evaluation of ices in solar-mass systems, a survey in the infrared wavelength range where 41 low-luminosity YSOs were included was conducted by using the infrared spectrograph on the Spitzer Space Telescope (Boogert et al. 2008).

The scientific scope of this study is to look for spatial variations in the water ice band profile at $3.08 \mu \mathrm{m}$ within a T Tauri star to detect possible, regional differences in water ice evolution. In Sects. 2 and 3, we scrutinize in detail our spectroscopic observations and data reduction, respectively. The derived water ice profiles are presented in Sect. 4. The ice profiles that could be derived are analyzed in Sect. 5.2 by considering both the absorption profiles of ice grains of different size and crystallization degree that are measured in laboratory. This well-established approach of deconvolution is described in Sect. 5.1. The question of whether evolved, i.e., large and/or crystyllized ice grains may finally be found is discussed in Sects. 5.3 and 5.4. We conclude this study with a summary in Sect. 6.

\section{Observations}

Our observations with the near-infrared (NIR) spectrometer CONICA (Lenzen et al. 2003) were performed at the $8.2 \mathrm{~m}$ YEPUN telescope of the Very Large Telescope (VLT) in spring and summer 2006. We used the L54_1_SL mode where a

\footnotetext{
2 The $L$ band covers a wavelength interval between $2.8 \mu \mathrm{m}$ and $4.2 \mu \mathrm{m}$ in the near-infrared range.
}

wavelength range of between $2.60 \mu \mathrm{m}$ and $4.20 \mu \mathrm{m}$ is covered. Observations with CONICA are supported by the adaptive optics NAOS (Rousset et al. 2003). A linear, spectral dispersion of $3.16 \mathrm{~nm} /$ pixel could be reached. The spatial resolution that could theoretically be reached was $0.054^{\prime \prime}$. Depending on the weather conditions during our observations, the pixel scale was $\sim 0.12^{\prime \prime}$. The slit of the spectrometer had a width of $0.172^{\prime \prime}$ and a length of $28^{\prime \prime}$.

We selected YSOs with quasi edge-on disks to be able to observe optically thick regions that shield ice from hard irradiation. Images, polarimetric maps, and previous object models were used as selection criteria. A visual extinction of $A_{\mathrm{V}}>3 \mathrm{mag}$ is sufficient for an effective shielding of water ice against irradiation (Murakawa et al. 2000).

A lunar occultation observation of YLW 16 A at $2 \mu \mathrm{m}$ (Simon et al. 1987) showed that the object is a single extended source and strongly inclined. Two brightness peaks are separated by $\sim 0.5^{\prime \prime}$ in a $K$ band image obtained by NICMOS at the Hubble Space Telescope (HST; Allen et al. 2002). These peaks are assigned to the optically thin bipolar envelope.

Table 1 provides an overview of our observations of the target YLW 16 A and the corresponding standard star HR 6070. The brightness of the standard star, an A $0 \mathrm{~V}$ star, is known from the standard star catalog of van Bliek et al. (1996). For the preparation of the observations, the $L$ band brightness of the target was estimated in this study by considering the $H$ band magnitudes and the $(H-K)$ and $(K-N)$ colors of additional YSOs of the same star-forming region (Allen et al. 2002).

Our target was observed using the nodding mode with a nodding angle of $10^{\prime \prime}$. Thus, the target was successively observed in different detector areas where the sky background could be eliminated by subtracting subsequent exposures. The chopping mode, which provides a more effective elimination of short-term variations in the sky, was not available in the observation mode used.

The target was observed twice, with two orthogonal orientations of the slit. The observation sequence started with a slit orientation parallel to the rotational axis of the object (position: p-parallel). In a second exposure, the slit was rotated by $90^{\circ}$ (position: o-orthogonal). The photometric center served as a rotation center. This procedure is assumed to allow the determination of the spatial distribution of water ice in the different regions of the circumstellar environment.

In the appendix of this publication, we list additional observations of YSOs within this program. These YSOs also belong to the $\rho$ Ophiuchi region.

\section{Data reduction}

When reducing the spectroscopic data obtained with NAOSCONICA, particular problems have to be taken into account. The specific steps of the reduction are therefore considered in detail:

i. Bad pixels are localized and replaced by the median of the pixels within the surrounding $3 \times 3$ detector segment.

ii. The background of detector, telescope, and sky are eliminated by the subtraction of successive exposures for different nodding positions. Owing to unavailability of the chopping mode that can clear short-term variations in the sky, telluric features cannot be completely removed in the object spectra. The resulting images are flatfielded.

iii. The spatial and spectral dimensions of a spectroscopic exposure are assigned to the rows and columns of the image matrix, respectively, to a first approximation. A Gaussian 
Table 1. Overview of our observations with NAOS-CONICA.

\begin{tabular}{|c|c|c|c|c|c|c|c|c|}
\hline Object & $\alpha(\mathrm{J} 2000)$ & $\delta(\mathrm{J} 2000)$ & $L[\mathrm{mag}]$ & $F W H M\left[{ }^{\prime \prime}\right]$ & Date & $U T$ & $A M$ & $T_{\text {tot }}$ \\
\hline HR 6070 & 161817.9 & -283650 & 4.807 & - & April 28th, 2006 & $7: 00-7: 02$ & 1.0 & $2.0 \mathrm{~s}$ \\
\hline YLW 16 A (p) & 162728.0 & -243934 & 6.7 & 0.5 & April 28th, 2006 & $7: 26-8: 08$ & 1.0 & $60 \times 3 \times 4.0 \mathrm{~s}$ \\
\hline YLW 16 & 162728.0 & -243934 & 6.7 & 0.5 & August 10th, 2006 & $0: 45-2: 00$ & 1.1 & $26 \times 50 \times 2.8 \mathrm{~s}$ \\
\hline HR 6070 & 161817.9 & -283650 & 4.807 & - & August 10th, 2006 & $2: 51-2: 59$ & 1.3 & $2.0 \mathrm{~s}$ \\
\hline
\end{tabular}

Notes. Coordinates (Cutri et al. 2003), L band magnitude, spatial FWHM, date, observing time (UT), as well as the mean airmass (AM) during the observations are listed. The object YLW 16 A was observed twice using two different slit orientations: parallel (p) and orthogonal (o) to the rotational axis of the YSO. The quantity $T_{\text {tot }}$ is the total exposure time. Directly before or after the observation, the standard star HR 6070 was observed.

function is fitted to each row of the array to determine the maximum of the spatial intensity distribution. The counting rates of the pixels within $4 \times F W H M$ (full width at half maximum) of each Gaussian function are added. Alternatively, the Gaussian functions are divided into seven single intervals resulting in spatially adjacent spectra. For a pixel scale of $0.12^{\prime \prime}$ and the source distance of $160 \mathrm{pc}$, the single intervals have widths of about $20 \mathrm{AU}, 20 \mathrm{AU}, 30 \mathrm{AU}$, and $40 \mathrm{AU}$, respectively, symmetrically arranged of increasing width at increasing distance from the brightness maximum. The interval of the central region has a width of $20 \mathrm{AU}$, the intervals of the outermost regions have widths of $40 \mathrm{AU}$.

iv. The wavelength calibration of the $L$ band spectra acquired with NAOS-CONICA is difficult because observations of specific calibration lamps could not be performed in this observing mode. Intrinsic stellar emission lines such as the Pf $\gamma$-line at $3.74 \mu \mathrm{m}$ (Wallace \& Hinkle 2002) do not clearly emerge from the background noise. Therefore, the measured spectra were cross-correlated with the telluric features of a sky spectrum obtained from $\mathrm{NSF} / \mathrm{NOAO}^{3}$. As the shapes of the asymmetrically curved telluric lines are not known, the accuracy of the wavelength calibration cannot be better than the accuracy provided by a pixel width.

v. To perform subsequent flux calibration, the standard star HR 6070 was observed in the immediate vicinity $\left(\sim 1^{\circ}\right)$, directly before or after the observation of the target. This ensured that instrumental and the atmospheric transmission could be determined, simultaneously. The division by a black-body function $B_{v}\left(T_{\text {eff }}\right)$ with $T_{\text {eff }}=9980 \mathrm{~K}$ for HR 6070 (Allen 2000) helps to reduce the influence of the continuum of the standard star. Considering the template spectrum of an F 0 V star in $L$ band (Rayner et al. 2009), the template spectrum of an A $0 \mathrm{~V}$ star up to $3.0 \mu \mathrm{m}$ (Pickles 1998) and the amplitude of the absorption depth of the water ice feature, potential stellar lines are assumed to be neglected, i.e., we do not correct for potential absorption lines in the spectrum of HR 6070. Figure 1 presents the normalized transmission of sky and instrument that were obtained from the observation of the standard star HR 6070. Spectral shifts of the standard star spectra on a sub-pixel scale allowed an improved elimination of the telluric lines in the spectrum of the target. To remove atmospheric residuals and data points affected by poor atmospheric transmission, sections of the spectra with less than $35 \%$ transmission are not considered in the analysis. These vary from exposure to exposure depending on weather conditions.

\footnotetext{
3 The sky spectrum is available at http://www. eso.org/sci/facilities/paranal/ instruments/.
}

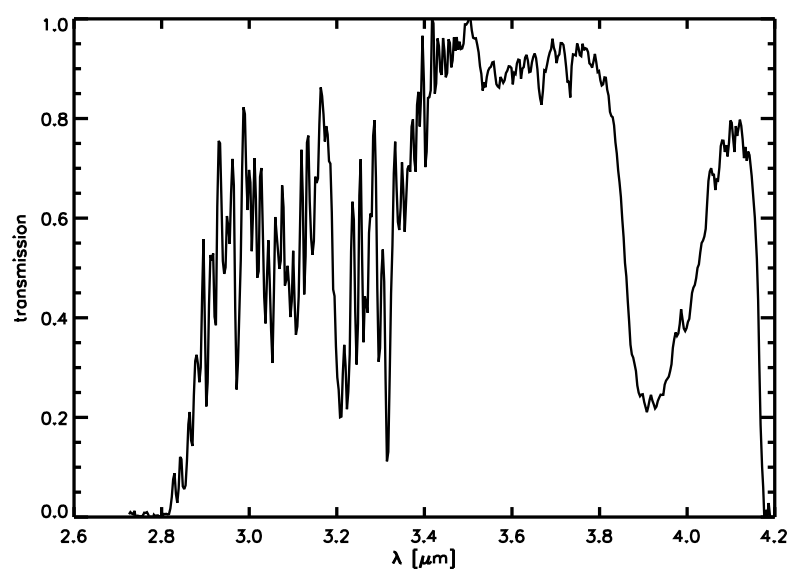

Fig. 1. Transmission of atmosphere and instrument derived from the spectrum of the standard star HR 6070.

vi. By considering the photometric $L^{\prime}$ band spectra ${ }^{4}$ and the filter curves that were formerly used to perform the photometric measurements of the standard star (van Bliek et al. 1996), a photometric flux calibration of the observed spectra of the target is possible. We note that the resulting scale factor of the photometric calibration can vary by more than $15 \%$ during a night (Przygodda 2004).

\section{Results}

Figure 2 shows the resulting $L$ band spectra of the target for which the counting rates of $4 \times F W H M$ of the spectrum were determined (Sect. 3). To provide spatial information, Fig. 3 shows the spectra derived from seven spatially adjacent stripes of the total spectra measured in orthogonal orientations. Spectra 1 to 7 represent spectra derived from source regions with projected widths of $40 \mathrm{AU}, 30 \mathrm{AU}, 20 \mathrm{AU}, 20 \mathrm{AU}, 20 \mathrm{AU}, 30 \mathrm{AU}$, and $40 \mathrm{AU}$, respectively.

The NICMOS/HST-images of the class-I-object YLW 16 A exhibit a bipolar envelope above and below its circumstellar disk (Allen et al. 2002). Both bipolar components yield two separate spectra I and II in the (p)-orientation of the slit.

The photometric flux can be compared to photometric measurements of the Infrared Array Camera (IRAC) onboard of the Spitzer satellite. We perform photometry on the pipeline mosaic available at the Spitzer archive ${ }^{5}$. The photometric calibration of IRAC is based on an aperture whose radius is set to be 10 pixels.

\footnotetext{
${ }_{4}$ The $L^{\prime}$ band covers the NIR range between $3.4 \mu \mathrm{m}$ and $4.2 \mu \mathrm{m}$.

5 http://irsa.ipac. caltech.edu/Missions/spitzer.html
} 

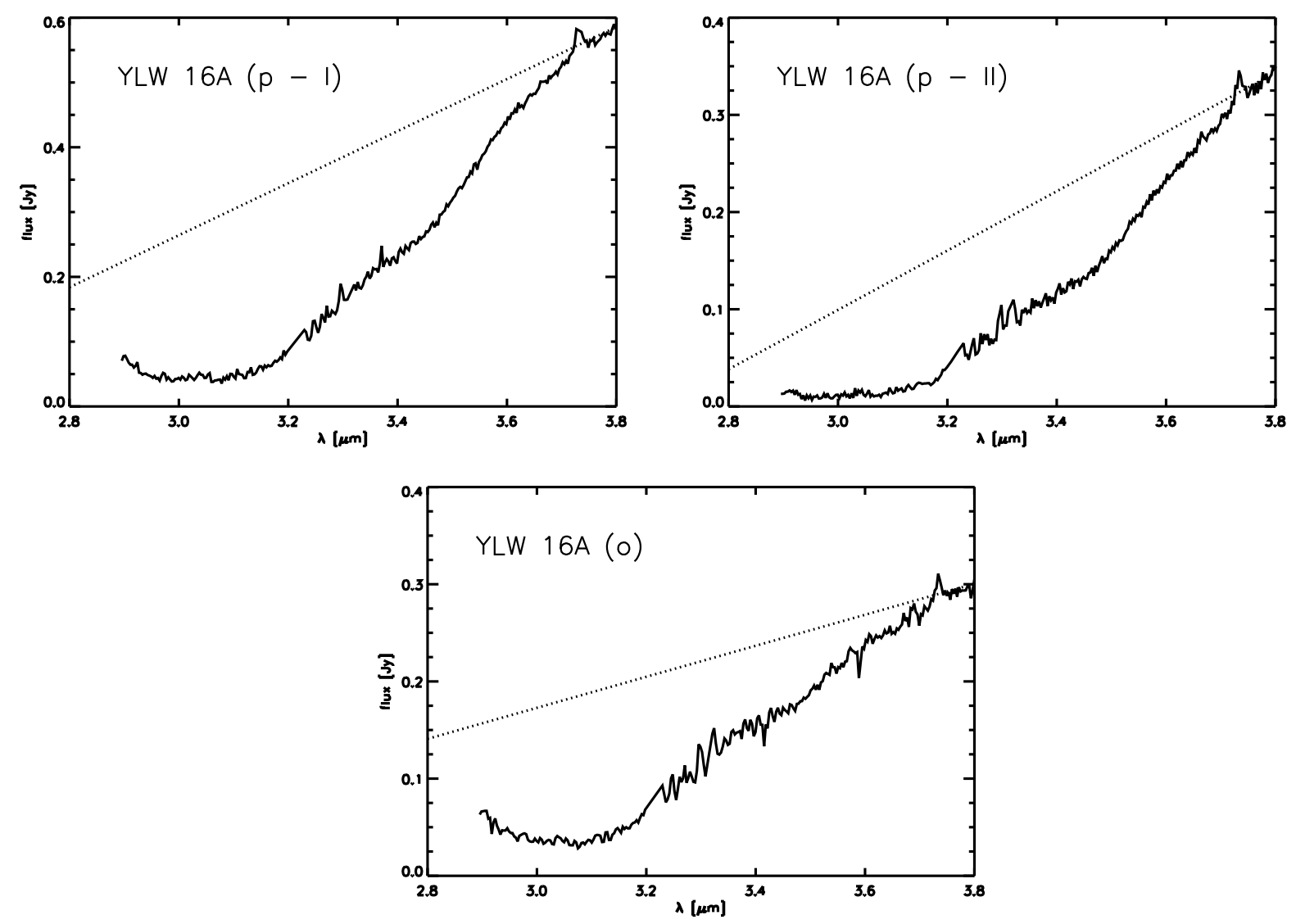

Fig. 2. Extracted and photometrically calibrated $L$ band spectra of the source YLW 16 A for slit orientations parallel and orthogonal to its rotational axis. The counting rates of $4 \times F W H M$ of the (spatial) widths of the spectra were determined. The dotted lines represent the "best-fit" spectral continua that we assume in our modeling approach.

The flux amounts to $0.79 \mathrm{Jy}$ at $3.6 \mu \mathrm{m}$ after background subtraction. In contrast to NAOS-CONICA, IRAC does not allow us to spatially resolve the geometrical structure of YLW 16 A. Therefore, the different spatial resolution powers of IRAC and NAOS-CONICA explain the differences between the $L$ band fluxes.

All spectra derived from our NAOS-CONICA observations display the broad water-ice band absorption feature with its minimum at $\sim 3.1 \mu \mathrm{m}$. As the atmospheric transmission is low at wavelengths of $<3.0 \mu \mathrm{m}$ (Fig. 1), the water ice feature is truncated at this lower wavelength end of the spectra.

\section{Modeling of the water ice band}

\subsection{Modeling approach}

By definition, the depth of the absorption band is the natural logarithm of the ratio of the measured flux $F_{\text {obs }}$ to the continuum flux $F_{\text {cont }}$ above the absorption band. It depends on the path $L$ through the absorbing material, its (mean) extinction $Q_{\text {ext }}$, and the particle density $\rho$. Whittet (2002) derived the optical depth $\tau$ of spherical particles with a mean radius $a$ and a mean material density $\eta$ to be

$$
\begin{aligned}
\tau & =\ln \left(\frac{F_{\mathrm{obs}}}{F_{\mathrm{cont}}}\right) \\
& =L \rho \frac{Q_{\mathrm{ext}} \pi a^{2}}{4 / 3 \pi a^{3} \eta}=L \rho \times \kappa_{\mathrm{ext}}=K_{0} \sum_{i}\left(K_{i} \times \kappa_{\mathrm{ext} ; i}\right) .
\end{aligned}
$$

The quantity $\kappa_{\mathrm{ext}}$ represents the (total) mass extinction. The extinction results from a linear superposition of mass extinctions $\kappa_{\text {ext; } i}$ of material $i$ that are weighted with the coefficient $K_{i}$ (Martin 1978; Šolc 1980). The quantity $K_{0}=L \rho$ is a constant for each object.

To determine the ice components that effectively contribute to the $3 \mu \mathrm{m}$-absorption band, extinction profiles of ice grains of different size and crystallinity are linearly combined to fit the ice profile. As a first approximation, scattering effects can only be neglected if the condition

$$
\frac{2 \pi a}{\lambda}<\frac{1}{|m|}
$$

is fulfilled (Dartois et al. 2001), where the quantity $|m|$ is the complex refractive index. For $|m| \approx 1.35$ (Querry et al. 1969) and $\lambda=3.5 \mu \mathrm{m}$, we obtain $a<0.41 \mu \mathrm{m}$. This result justifies the consideration of scattered radiation. Furthermore, the bipolar envelope of the object YLW 16 A appears in scattered light in the corresponding $K$ band images obtained with NICMOS/HST. In contrast to the absorption profiles, the extinction profiles are asymmetric, i.e., more bell-shaped at longer wavelengths (Dartois \& d'Hendecourt 2001).

For our fitting routine, we assume opacities of entirely amorphous $(T=10 \mathrm{~K})$ and crystallized $(T>140 \mathrm{~K})$ water ice. The ice grain radii $a$ are $0.1 \mu \mathrm{m}, 0.3 \mu \mathrm{m}, 0.5 \mu \mathrm{m}, 0.8 \mu \mathrm{m}$, and $1.5 \mu \mathrm{m}$. In this study, grains with $a \geqq 0.5 \mu \mathrm{m}$ and $a<0.5 \mu \mathrm{m}$ are called large and small grains, respectively. A restriction to two grain radii (e.g., $0.1 \mu \mathrm{m}$ and $0.8 \mu \mathrm{m}$ ) only increases the deviation between model and measurement but does not modify 
A. A. Schegerer and S. Wolf: Crystallized water ice in T Tauri objects
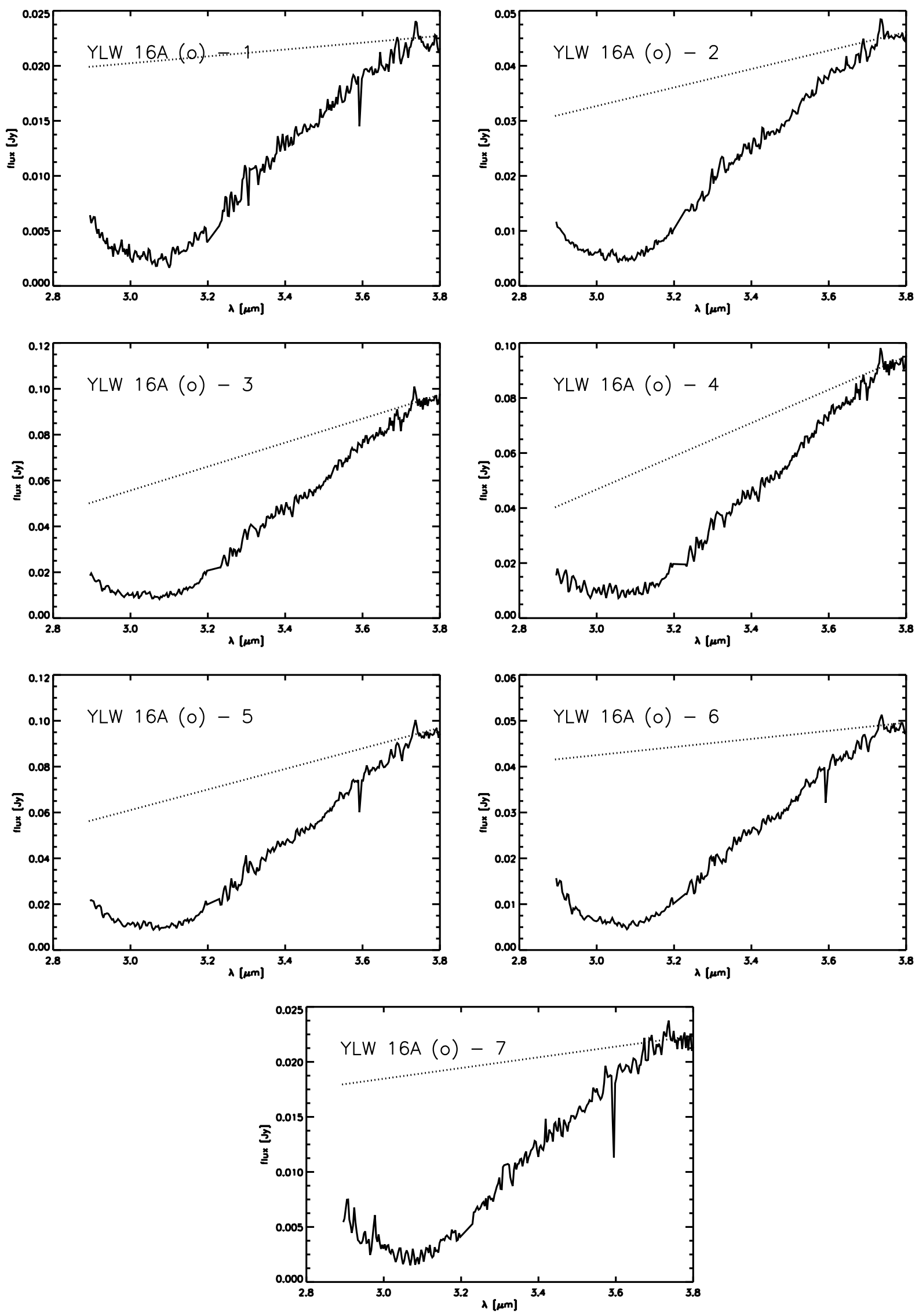

Fig. 3. Extracted and photometrically calibrated $L$ band spectra of YLW 16 A derived from seven spatially adjacent stripes cut from the acquired spectra. The spectra 1 to 7 represent spectra derived from source regions of widths $40 \mathrm{AU}, 30 \mathrm{AU}, 20 \mathrm{AU}, 20 \mathrm{AU}, 20 \mathrm{AU}, 30 \mathrm{AU}$, and $40 \mathrm{AU}$, respectively. 

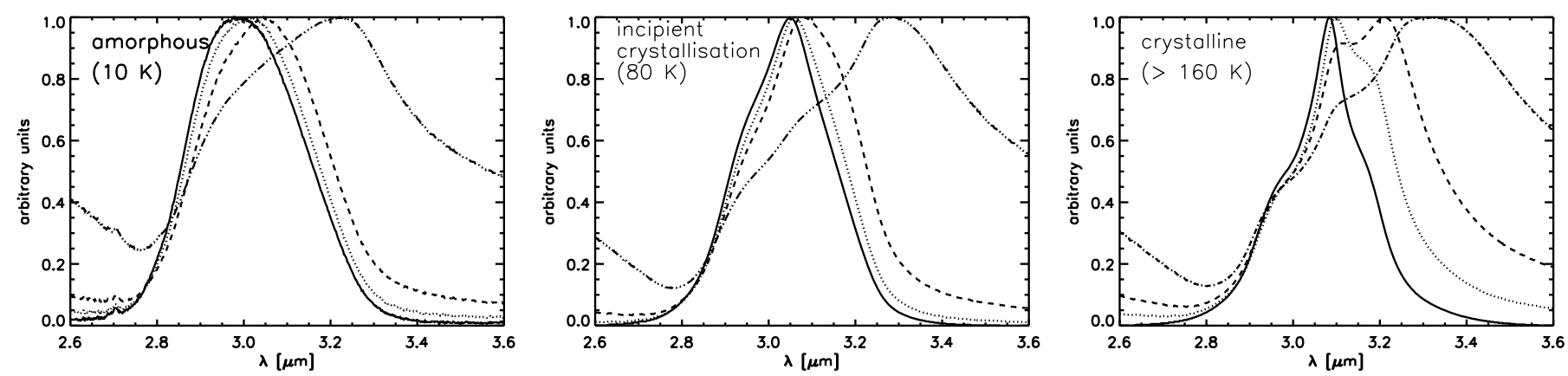

Fig. 4. Normalized extinction profiles $\kappa_{\text {ext } ; i}$ of amorphous (left panel) and crystallized (right panel) water ice for particle radii $a$ of $0.1 \mu \mathrm{m}$ (solid curve), $0.5 \mu \mathrm{m}$ (dotted line), $0.8 \mu \mathrm{m}$ (dashed curve), and $1.5 \mu \mathrm{m}$ (dot-dashed curve). The profiles were normalized by their maximum amplitudes. The middle panel shows the profile of water ice with a temperature of $80 \mathrm{~K}$ where crystallization starts. A shift of the maximum towards longer wavelengths is caused by grain growth and increasing crystallization degree.

the resulting mass ratios $K_{i}$. Figure 4 shows the mass extinction $\kappa_{\text {ext }}(\lambda)$ of amorphous and crystallized water ice used in our fitting approach (Eq. (1)). Assuming spherical, compact ice particles, the mass extinction curves are derived using the program MIEX (Wolf \& Voshchinnikov 2004), which is based on Mie scattering. This program calculates the extinction profiles by considering the grain size and refractive indices (Schmitt et al. 1998; Dartois \& d'Hendecourt 2001).

An unambigous determination of the continuum flux $F_{\text {cont }}$ above the absorption band is difficult. Owing to reduced atmospheric transmission (Fig. 1), parts of the spectra at shortwavelengths are missing. The determination of the continuum at longer wavelengths $(>3.6 \mu \mathrm{m})$ is also difficult because some telluric lines could not be removed entirely during data reduction, producing noisy spectra. In previous investigations of the water ice band (Thi et al. 2006; Dartois et al. 2002; Boogert et al. 2008), a Planck function $B_{v}(T)$ or a spline function was fitted to the continuum and - if available - to photometric data points in adjacent spectral bands to determine the continuum. In addition, studies were performed where the spectral energy distribution of the entire infrared wavelength range was fitted using disk models (Pontoppidan et al. 2004). Assuming a spatial resolution of $0.12^{\prime \prime}$ ( $\left.20 \mathrm{AU}\right)$ for our NAOS-CONICA observations, which is smaller than the true spatial extension of the object, only a fraction of the total flux of the disk is detected. Therefore, when comparing with IRAS observations (Sect. 4), photometric measurements do not have to represent the absolute level of the $L$ band spectra measured with NAOS-CONICA.

Apart from either the Planck function $B_{v}(T)$ or a spline function, we use a straight line to determine the continuum. The straight line is rotated successively around different spectral points resulting in different optical depths (Eq. (1)). The spectral points about which the line is rotated are the sampling points at $3.6 \mu \mathrm{m}$ and $3.8 \mu \mathrm{m}$, respectively. The latter point is used because the water ice band and other compounds (Sect. 1) do not contribute to the spectrum around $3.8 \mu \mathrm{m}$. The angular step used to represent the rotation depends on the depth of the absorption profile. Smaller angular steps were not found to improve the fit to the optical depth $\tau$. We found that the resulting optical depth $\tau$ is strongly affected by the gradient of the continuum's line. We finally look for the line at a certain rotational center whose corresponding linear combination of extinction profiles $\kappa_{\text {ext } ; i}$ reproduce the optical depth $\tau$ the most successfully, i.e., with the smallest reduced chi-square $\chi^{2}$ (Eq. (3)). We use the fitting routine presented in Schegerer et al. (2006). Representative continuum lines are drawn in the spectra shown in Figs. 2 and 3.
The determinations of both the continuum and the ice components that effectively contribute to the absorption profile were tested by analyzing the spectrum of the YSO GL 989. Its $L$ band spectral distribution was previously investigated by Dartois \& d'Hendecourt (2001). In contrast to our measurements, the source was formerly observed with the ISO satellite (Infrared Space Observatory) where the spectrum at short wavelengths could be retained. But when this spectrum is cut at short wavelengths up to a wavelength of $2.9 \mu \mathrm{m}$, the resulting spectrum corresponds to the NAOS-CONICA spectra that we acquired during different weather conditions. The results of the analysis of the $3 \mu \mathrm{m}$ spectra of GL 989 with and without the shortwavelength interval are finally compared. Potential differences can then be considered as an approximation of the systematic error caused by the missing interval of the NAOS-CONICA spectra up to $2.9 \mu \mathrm{m}$. We note that Dartois \& d'Hendecourt (2001) used a Planck function to determine the underlying continuum of GL 989 assuming a temperature of $T=500 \mathrm{~K}$. Furthermore, they considered extinction profiles of ice grains with a continous size distribution.

Figure 5 shows the results. Independent of the rotational center used, the fitting results obtained from the spectra that are truncated at $2.9 \mu \mathrm{m}$ differ by $<15 \%$ from the results derived from spectra for which the lower end of the $L$ band is considered. The mass contribution of crystallized ice can be neglected in all spectra. The mass contribution of amorphous, large grains depends strongly on the rotational center used. Both the non-truncated and truncated spectra exhibit only marginal evidence of large water ice grains $(<1 \%)$ when a rotational center at $3.8 \mu \mathrm{m}$ is used for the linear continuum. In contrast, the contribution of amorphous, large grains with a size of $0.5 \mu \mathrm{m}$ is $>85 \%$ when a rotational center at $3.6 \mu \mathrm{m}$ is used. The fitting results deviate by between $\chi^{2}=0.0015$ and $\chi^{2}=0.003$ from the measurement (Eq. (3)). The deviation from the measurement is larger for fits with the rotational center at $3.6 \mu \mathrm{m}$.

\subsection{Modeling results}

Table 2 and Figs. 6 and 7 summarize our modeling results. The quality of each fit is characterized by $\chi^{2}$ (e.g., Press et al. 1986) with

$\chi^{2}=\frac{1}{N_{\lambda}-f} \sum_{\mathrm{i}=1}^{\mathrm{N}_{\lambda}}\left(\mathcal{F}_{v}^{\text {model }}\left(\lambda_{\mathrm{i}}\right)-\mathcal{F}_{v}^{\text {data }}\left(\lambda_{\mathrm{i}}\right)\right)^{2}$. 

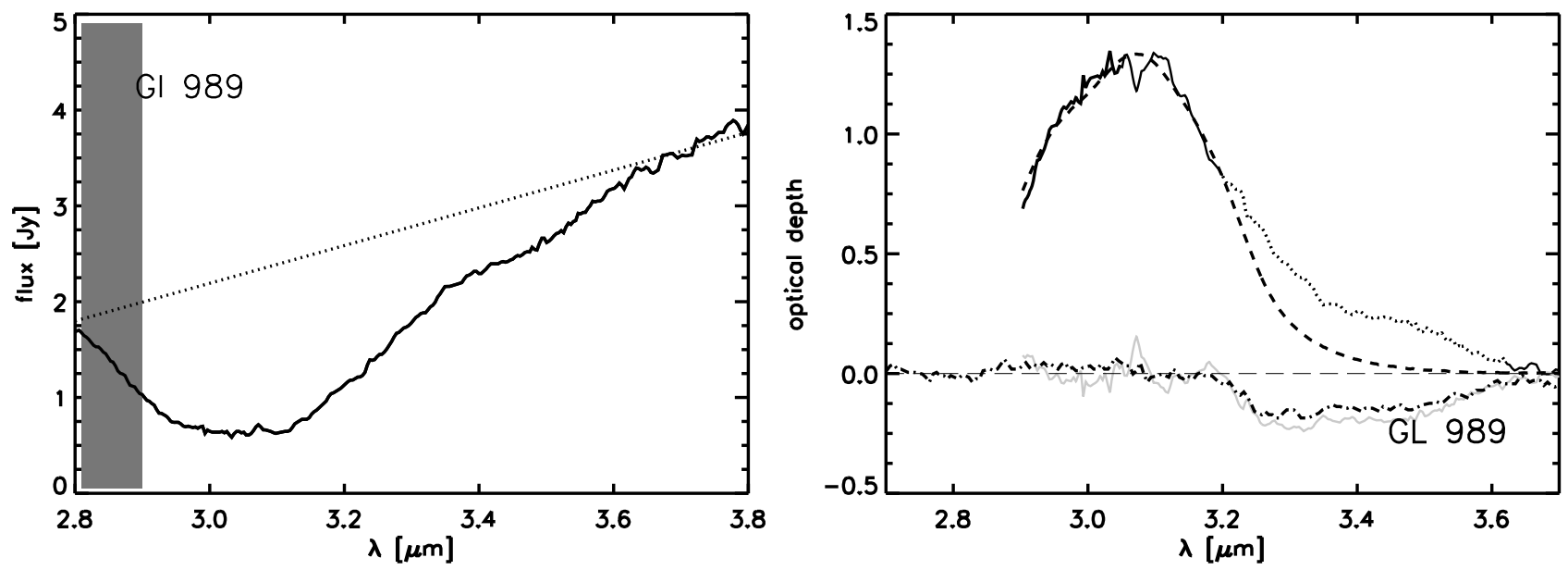

Fig. 5. Left panel: $L$ band spectrum of the object GL 989 measured with the ISO satellite. Because of the lower transmission of the sky, the spectral interval at short wavelengths that is indicated by gray color is difficult to observe using NAOS-CONICA or any other instrument on the ground because of the lower transmission of the sky. The dotted line represents the likely linear continuum.

Right: Derived optical depth $\tau$ of the water ice band of GL 989 truncated at $2.9 \mu \mathrm{m}$. The absorption between $3.2 \mu \mathrm{m}$ and $3.6 \mu \mathrm{m}$ that is not only caused by water ice, is not considered by the modeling (dotted curve). The linear combination of ice profiles $\kappa_{\text {ext } ; i}$ that represents the band with the smallest deviation from the measurement, i.e., smallest $\chi^{2}$, is plotted by the dashed curve. The thin gray curve represents the deviaton of the model from measured data. For comparison, the deviation for the modeling of Dartois \& d'Hendecourt (2001) is also plotted (dot-dashed curve). The long-dashed curve represents the zero level.

Table 2. Relative mass contribution of amorphous-small $\left(m_{\mathrm{a} ; \mathrm{s}}: 0.1 \mu \mathrm{m}\right.$ and $\left.0.3 \mu \mathrm{m}\right)$, amorphous-large $\left(m_{\mathrm{a} ; 1}: 0.5 \mu \mathrm{m}, 0.8 \mu \mathrm{m}\right.$, and $\left.1.5 \mu \mathrm{m}\right)$ and crystallized $\left(m_{\mathrm{c}}\right.$ : all particle radii) water ice grains derived from our modeling approach by using a rotational center at $3.8 \mu \mathrm{m}$.

\begin{tabular}{lccccccc}
\hline \hline Exposure & $P A\left[^{\circ}\right]$ & $N_{\mathrm{A}}\left[10^{22} \mathrm{~m}^{-2}\right]$ & $A_{\mathrm{V}}[\mathrm{mag}]$ & $m_{\mathrm{a} ; \mathrm{s}}[\%]$ & $m_{\mathrm{a} ; 1}[\%]$ & $m_{\mathrm{c}}[\%]$ & $\chi$ \\
\hline YLW 16 A (p - I) & 178 & 1.7 & 19 & 97 & - & 3 & 0.0074 \\
YLW 16 A (p - II) & 178 & 2.5 & 27 & $>99$ & $<1$ & $<1$ & 0.020 \\
YLW 16 A (o) & 88 & 2.2 & 24 & 70 & 1.7 & 28 & 0.078 \\
YLW 16 A (o) - 1 & 88 & 2.0 & 22 & 32 & 1.1 & 67 & 0.011 \\
YLW 16 A (o) - & 88 & 1.9 & 20 & 31 & 1.4 & 68 & 0.0046 \\
YLW 16 A (o) - - & 88 & 1.7 & 19 & 93 & 1.0 & 5.7 & 0.0042 \\
YLW 16 A (o) - 4 & 88 & 1.8 & 20 & 78 & 10 & 12 & 0.010 \\
YLW 16 A (o) - 5 & 88 & 1.7 & 19 & 87 & 4.9 & 7.8 & 0.0079 \\
YLW 16 A (o) - 6 & 88 & 2.0 & 22 & 84 & 7.6 & 8.2 & 0.0042 \\
YLW 16 A (o) - 7 & 88 & 2.0 & 22 & 4.5 & 3.2 & 92 & 0.0087 \\
\hline
\end{tabular}

Notes. The position angles $(P A)$ of the spectroscopic slit, measured from North to East, are additionally listed. The regions 1 to 7 represent spectra that originate in source regions with widths of $40 \mathrm{AU}, 30 \mathrm{AU}, 20 \mathrm{AU}, 20 \mathrm{AU}, 20 \mathrm{AU}, 30 \mathrm{AU}$, and $40 \mathrm{AU}$. The spectra 3-5 originate closer to the photocenter than the spectra $1,2,6$, and 7 . In contrast, for the first three exposures listed, the counting rates within $4 \times F W H M$ of the spatial dimension are summarized. The quantities $N_{\mathrm{A}}$ and $A_{\mathrm{V}}$ represent the column density of water ice particles and the visual extinction derived along the line of sight (Eqs. (4), and (5)).

The data with the largest $\chi^{2}$ have the lowest signal-to-noise ratios. In Eq. (3), $N_{\lambda}$ is the number of data points, $f$ the degree of freedom, and $\mathcal{F}_{v}^{\text {model }}(\lambda)$ and $\mathcal{F}_{v}^{\text {data }}(\lambda)$ are the modeled and measured fluxes, respectively.

The features of additional compounds (e.g., of ammonia hydrate) is superimposed on fractions of the ice absorption band at a wavelength of $\sim 3.4 \mu \mathrm{m}$. The spectral region between $3.25 \mu \mathrm{m}$ and $3.65 \mu \mathrm{m}$ is not considered by our modeling approach (dotted curves).

In contrast to our results for spectra with the parallel slit orientation of the slit, a larger amount of crystalline grains $(28 \%)$ contributes to the spectrum of the orthogonal slit orientation (3rd line in the Table 2). We point out that this spectrum can be fitted by a larger amount of large, i.e., $0.5 \mu \mathrm{m}$-sized amorphous grains, if crystallized grains are excluded from the fit. As the fit then worsens by $35 \%$, this finding can be considered as evidence of crystalline dust grains, as the maximum of the extinction profile of larger amorphous grains shifts to longer wavelengths similar to the shift of the extinction profile of crystalline grains.

In Fig. 7, the spectra 1 to 7 correspond to different regions within the source that could be spatially resolved by our observations with NAOS-CONICA for the orthogonal slit orientation (Sect. 3). Because of the too noisy data, we do not consider the optical depth derived from the observation where the spectroscopic slit was orientated parallel to the rotational axis. As we obtain tighter fits to the derived optical depth, i.e., smaller $\chi^{2}$, we focus only on the results obtained from fits where the continuum's line is rotated around $3.8 \mu \mathrm{m}$ but without forgetting the corresponding results obtained for a rotational center at $3.6 \mu \mathrm{m}$.

The spectra 3, 4, and 5 that were derived from the most central region of the source around the photocenter appear to contain the largest contribution of non-evolved, i.e., amorphous, small grains, while the spectra from the outer source regions exhibit a greater contribution from crystallized water ice. These crystalline, peripheral grains also contributes to the spectrum where 



Fig. 6. Optical depth $\tau(\lambda)$ derived from the spectra of Fig. 2 considering Eq. (1). Dotted curves represent the wavelength interval that is not considered in the modeling approach. The water ice band in the interval between $3.2 \mu \mathrm{m}$ and $3.6 \mu \mathrm{m}$ is superimposed on the absorption bands of ammonia hydrate and other compounds. The models are represented by dashed curves (Table 2). The gray curves around the zero level are deviations from the measured optical depth. The source is not spatially resolved in the optical depths shown here.

the counting rates of $4 \times F W H M$ were summarized (3rd line in the Table 2).

Considering Fig. 3 and the flux scales used, it is conspicuous that different gradients are found for fitting the continua of spectra that originate in different source regions. However, if steeper gradients were used to fit continua of spectra originating in outer source regions, even a larger contribution of crystallized grains would be found. On the other hand, a shallower gradient for the continua derived in the more central source regions would favor a larger contribution of small amorphous grains. In any case, the fit is poorer when gradients other than those are used.

The fit results obtained for spectra in which a rotational center at $3.6 \mu \mathrm{m}$ is used, have qualitatively the same increasing contribution of crystalline grains towards the outer regions $\left(m_{\mathrm{c}}=57 \%, 0.25 \%, 98 \%\right.$ at the regions 1,3 , and 7 , respectively). However, as already mentioned in Sect. 5.1, the contribution of amorphous, large (i.e., $0.5 \mu \mathrm{m}$-sized) grains has increased at the expense of amorphous, small grains, in particular in the more central regions 4 , and 5 , where on average $m_{\mathrm{a} ; 1} \approx 88 \%$.

The column density $N_{\mathrm{A}}$ of the absorbing ice material can be determined by an integrating of the extinction profiles of amorphous water ice that are fitted to the optical depth $\tau(\lambda)$. According to Whittet (2002), we obtain

$N_{\mathrm{A}}\left(\mathrm{H}_{2} \mathrm{O}[\right.$ ice $\left.]\right)=\int_{2.8 \mu \mathrm{m}}^{3.8 \mu \mathrm{m}} \frac{\tau(\lambda)}{A} \frac{\mathrm{d} \lambda}{\lambda^{2}}$.

The integral extinction cross-section $A$ of amorphous water ice at a temperature of $T=10 \mathrm{~K}$ is $A=2.0 \times 10^{-18} \mathrm{~m} / \mathrm{molecule}$
(Hagen \& Tielens 1981b). As the O-H stretching mode is very strong and dominates over other species (Tielens et al. 1984), the $3 \mu \mathrm{m}$ band provides an accurate water ice column density. Teixeira \& Emerson (1999) found the following empirical relation between the column density $N_{\mathrm{A}}\left(\mathrm{H}_{2} \mathrm{O}[\right.$ ice] $)$ and the visual extinction $A_{\mathrm{V}}$ :

$N_{\mathrm{A}}\left(\mathrm{H}_{2} \mathrm{O}[\right.$ ice $\left.]\right)=\left(A_{\mathrm{V}}-2.1\right) \times 10^{21} \mathrm{~m}^{-2}$.

When considering spectra obtained from different source regions, the quantities $N_{\mathrm{A}}$ and $A_{\mathrm{V}}$ are higher by $18 \%$ and $16 \%$, respectively, in spectra obtained from outer than for those from the inner regions.

\subsection{Water ice growth in the disks around T Tauri stars?}

The spectra of central source regions derived from a rotational center of the continuum's line at $3.8 \mu \mathrm{m}$ show profiles of amorphous water ice grains that exhibit only weak hints of grain growth (Table 2). However, when considering the same results for a rotational center at $3.6 \mu \mathrm{m}$, the contribution of the absorption profiles of $0.5 \mu \mathrm{m}$-sized grains is significantly higher although the fit worsens by $24 \%$ up to $32 \%$. From Eq. (2), we find that scattered radiation is present only for grain sizes of up $\sim 0.5 \mu \mathrm{m}$. If we replace the extinction profiles of large grains ( $a>0.5 \mu \mathrm{m}$ ) with the corresponding absorption profiles, the spectroscopic contribution of large grains increases by only a few percent. 
A. A. Schegerer and S. Wolf: Crystallized water ice in T Tauri objects
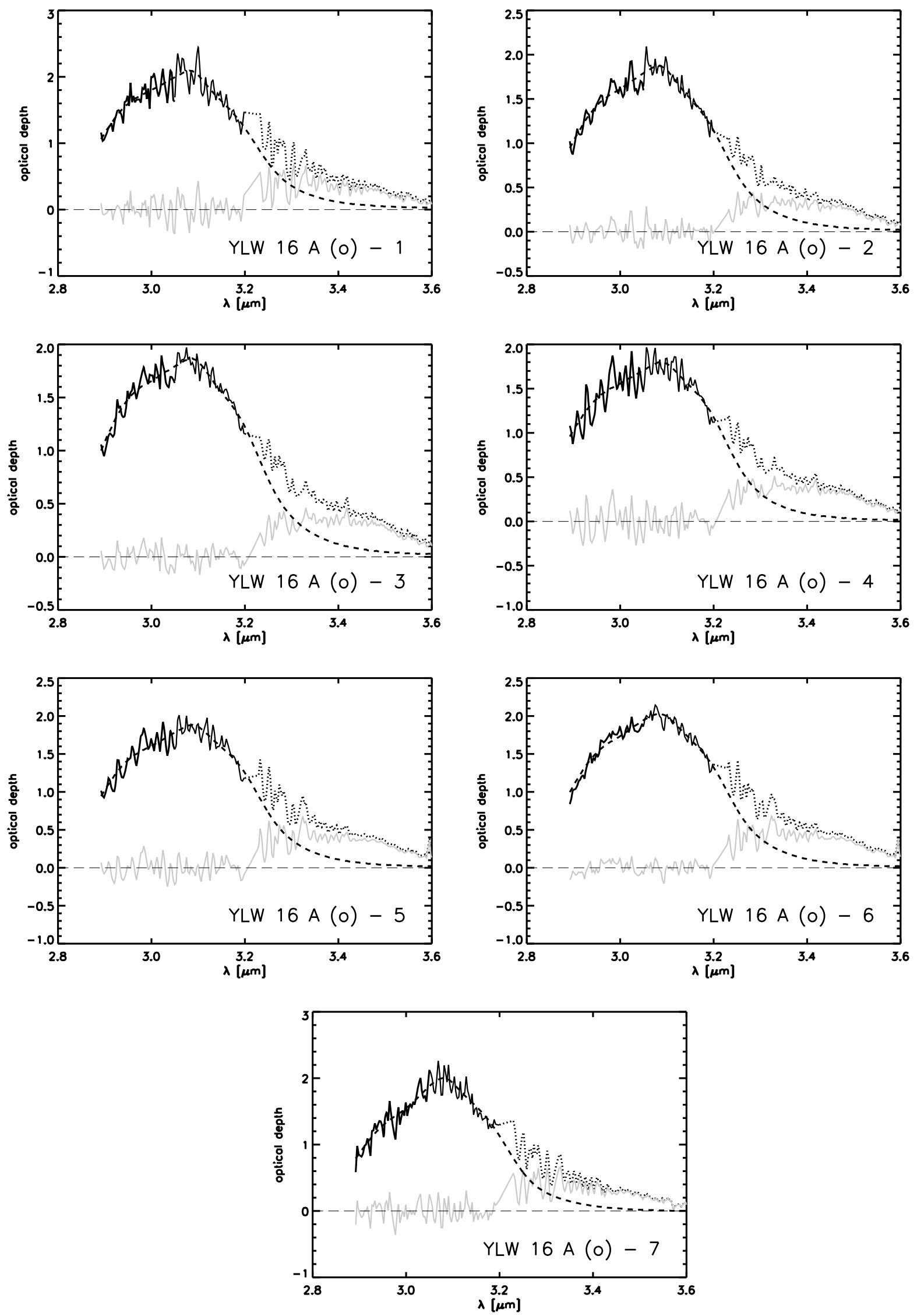

Fig. 7. Continuation of Fig. 6 but the spectra 1 to 7 correspond to different regions within the source that could be spatially resolved by our observations with NAOS-CONICA (Sect. 3). 
In previous studies, water ice grains larger than $1 \mu \mathrm{m}$ were not detected in YSOs. Thi et al. (2006) point to broadened water ice profiles in the spectra of several YSOs in the molecular cloud Vela indicating ice grain growth. There is at least one among five objects in their sample where the absorption profile of $0.5 \mu \mathrm{m}$ sized, amorphous ice grains can be used for modeling. Whether this result and the other evidence we have found indicate ice grain growth remains to be discussed in the context of the following issues. As shown in Schegerer et al. (2006), increasing the porosity of grains with constant radii broadens the $10 \mu \mathrm{m}$ silicate feature. We assume that porous water ice grains show similar behavior but this assumption can only be confirmed by future, theoretical studies. Furthermore, it is often assumed that ice grains have cores, such as those of $0.1 \mu \mathrm{m}$-sized, amorphous silicate (e.g., Jones \& Merrill 1976). The core serves as a seed where water is adsorbed. As shown in Fig. 8, a growth in the silicate core also results in a broadening of the $3 \mu \mathrm{m}$ absorption band (e.g., Smith et al. 1988). A broadening of the extinction profile can also be evoked by the shape of the continuum curve derived. In our study, the continuum is represented by a straight line, while the Planck function or spline functions are used in other studies. The Planck function, which is concave down, implies that there has been a broadening of the water ice band at long wavelengths, which automatically indicates ice grain growth. Finally, it is not clear to us why only $0.5 \mu \mathrm{m}$-sized grains are found when large dust grains are used in the fit. It has to be clarified whether the ice grain growth to sizes $a>0.5 \mu \mathrm{m}$ is physically prevented in YSOs or if this finding can be ascribed to the fitting approach used.

We conclude that an unambiguous identification of grain growth is difficult to make. In contrast, the discrimination between crystallized and amorphous grains should be easier as argued by Dartois \& d'Hendecourt (2001), as the extinction profile of crystallized ice narrows.

\subsection{Crystallized water ice in the disks around T Tauri stars?}

Because of the low temperatures and weak shielding of the interstellar media against hard irradiation, we assume that there is a negligible contribution of crystallized water ice when YSOs start to form from molecular clouds. The finding of crystallized water ice in YLW 16 A is now discussed.

As described in Sect. 3, information about the spatial ice distribution within the circumstellar environment can be extracted from the spectra measured with NAOS-CONICA. Instead of summarizing the intensities of the aperture, the Gaussian function that is fitted to the intensity distribution in each row of the image is symmetrically divided in steps of $20 \mathrm{AU}, 20 \mathrm{AU}$, $30 \mathrm{AU}$, and $40 \mathrm{AU}$ starting at the photocenter of the source. Independently of the rotational center used, we mainly find amorphous water ice close to the photocenter $\left(m_{\mathrm{a}}>88 \%\right)$. However, for increasing distance from the photocenter, the mass contribution of crystallized ice strongly increases (Table 2, Fig. 7).

Figure 9 shows the normalized optical depths derived from the depth of the peripheral region 7 (gray curve) that is overlaid on the mean of the depths of more central regions. The optical depths of the more central regions are lower than those of the peripheral regions. We note that if only amorphous grains are used in our deconvolution approach, the fitting worsens by up to $14 \%$ according to the parameter $\chi^{2}$ (Eq. (3); Table 2) derived.

The sketch in Fig. 10 shows a circumstellar disk where the location of amorphous and crystallized water ice grains are plotted considering our fitting results. We assume that the

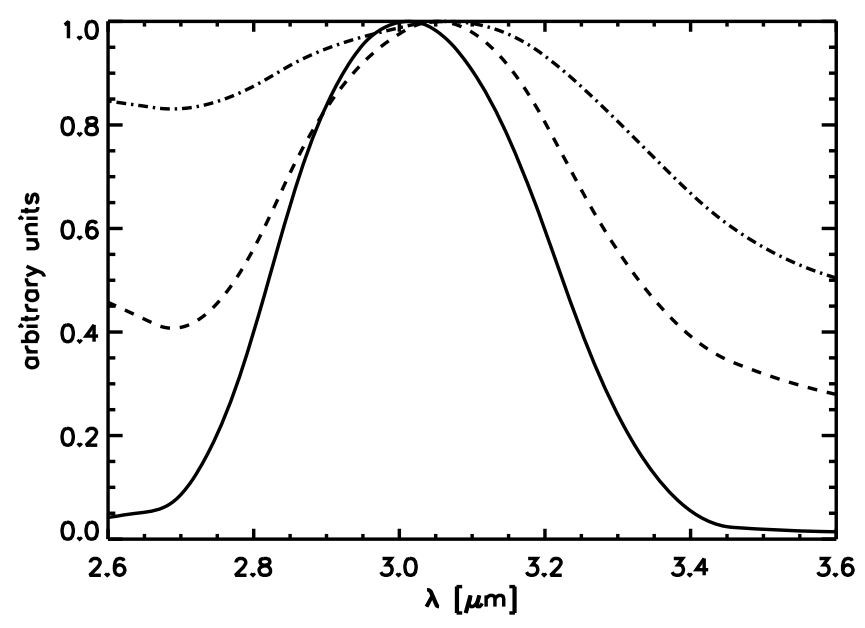

Fig. 8. Relation between extinction profile and the radius of the grain core. The core consists of amorphous silicate, while water ice forms a compact shell. The particles shown here have a water ice core with a radius of $0.1 \mu \mathrm{m}$ (solid curve), $0.5 \mu \mathrm{m}$ (dashed curve), and $0.8 \mu \mathrm{m}$ (dotdashed curve). The thickness of the ice shell is $0.1 \mu \mathrm{m}$. The profiles were normalized by the maximum value.

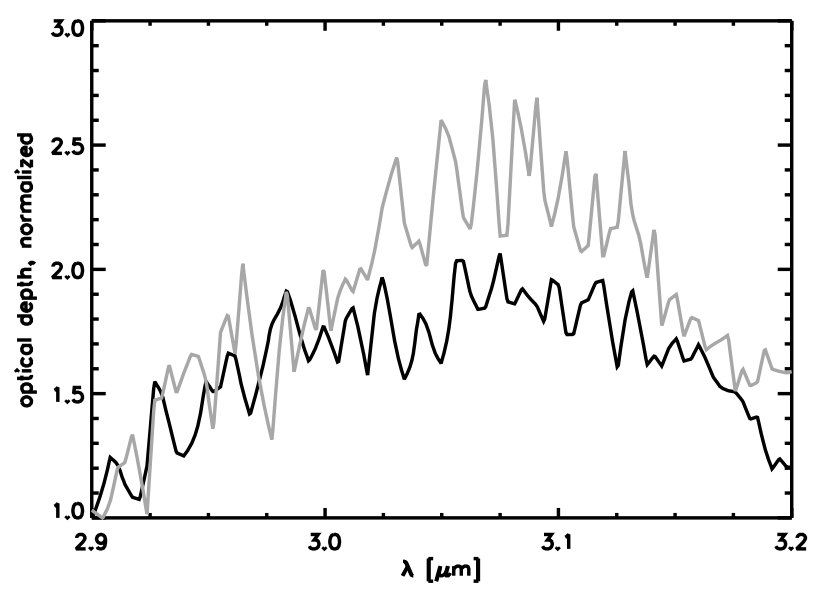

Fig. 9. Optical depths derived from the central (black curve) and peripheral (gray curve) regions of the YSO observed. The optical depths of the region 3,4 , and 5 were averaged to obtain the black curve while the depth from the region 7 was used to obtain the gray curve. For comparison, the curves are normalized at the optical depth at $\sim 2.9 \mu \mathrm{m}$. A larger amount of crystalline ice grains contribute to the narrow gray curve with a maximum at $3.075 \mu \mathrm{m}$. Almost only amorphous dust grains contribute to the black curve derived from the more central region.

crystallized water ice component has its origin in the upper disk layers at large disk radii, where water ice does not evaporate and is protected by the surrounding material of the circumstellar envelope from irradiation. Because of the higher temperatures, water ice at smaller radii close to the snowline is assumed to be crystallized inside the more embeded disk layers. The observation of the outer parts of the optically thick disk also explains the higher column density $N_{\mathrm{A}}$ inferred from spectra that originates in the outer regions (Table 2). Again, this finding does not depend on the rotational center used. The photocenter of the spectra is assumed to represent the optically thin bipolar envelope of the source observed with NICMOS/HST (Allen et al. 2002). Because of the low effective shielding in this central region, crystallized water ice is assumed to be destroyed by irradiation. 


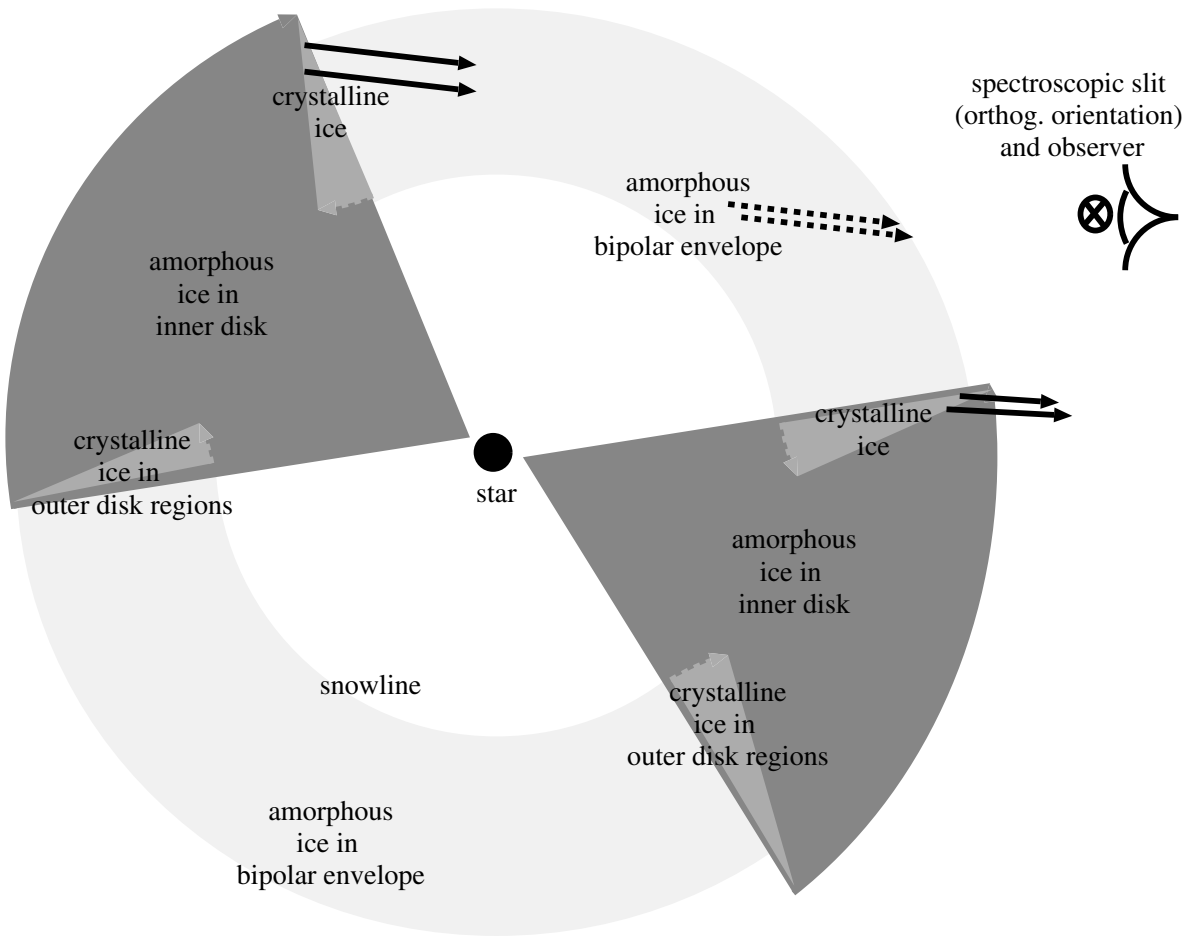

Fig. 10. Sketch for a strongly inclined circumstellar disk such as YLW $16 \mathrm{~A}$. We assume that amorphous, non-evolved water ice is located in the circumstellar disk and in the bipolar envelope. The assumed location of crystallized water ice in the disk is close to upper disk layers, at disk radii that are larger than that of the snowline. The observer and the spectroscopic slit are also sketched. The orthogonal slit orientation is shown. Radiation from amorphous and crystallized ice grains are represented by dashed and solid arrows, respectively.

The YSOs that were additionally observed with NAOSCONICA and whose observations are presented in the Appendix of this publication, do not show any evidence of crystallization. However, the spectra of these objects contain the water ice absorption feature. The question of whether these features originate in the circumstellar environment of these objects cannot unequivocally be answered here.

\section{Summary}

We have presented an $L$ band spectroscopic observation performed with NAOS-CONICA of a YSO that is strongly inclined. These systems allow the study of optical thick circumstellar regions that shield water ice from hard irradiation and evaporation. The absorption feature of water ice at $3.08 \mu \mathrm{m}$ was identified in the spectra with optical depths of between 1.8 and 2.5 , depending on the region observed. The optical depth, the water-ice column density, and the visual extinction are higher in the more peripheral regions at greater distance from the photocenter of our observations. The optical depths were derived from the spectra by assuming that a straight line represents the continuum flux.

Considering the extinction profiles of amorphous and crystallized water ice grains with sizes ranging from $0.1 \mu \mathrm{m}$ up to $1.5 \mu \mathrm{m}$, the derived optical depths were deconvolved using a fitting routine that was already presented in Schegerer et al. (2006). The optical depths of the spatially unresolved spectra were found to be dominated by the extinction profile of small, amorphous (i.e., non-evolved) water ice grains. However, in spectra for which the T Tauri object YLW 16 A could be spatially resolved, crystallized water ice could be found in possible outer disk regions, i.e., in disk layers at radii $>30 \mathrm{AU}$ in projection. The putative bipolar envelope of this source was not found to exhibit any evidence of crystallized material (Fig. 10). A growth in the size of the water ice grains cannot unambiguously be determined because the corresponding modification of the water ice feature may also be either caused by an increase in the porosity and/or a growth of the particle core.
Acknowledgements. We thank D. Semenov and M. Goto, in particular, for fruitfull discussions and their further support. Thanks are also due to J. Bouwman for his valuable assistance during the proposal preparation phase.

\section{References}

Allen, C. W. 2000, in Astrophysical Quantities, ed. A. N. Cox (Springer) Allen, L. E., Myers, P. C., Di Francesco, J., et al. 2002, ApJ, 566, 993 Bernstein, M. P., Elsila, J. E., Dworkin, J. P., et al. 2002, ApJ, 576, 1115 Boogert, A. C. A., Pontoppidan, K. M., Lahuis, F., et al. 2004, ApJS, 154, 359 Boogert, A. C. A., Pontoppidan, K. M., Knez, C., et al. 2008, ApJ, 678, 985

Bouwman, J., Meeus, G., de Koter, A., et al. 2001, A\&A, 375, 950

Chen, W. P., \& Graham, J. A. 1993, ApJ, 409, 319

Chiang, E. I., Joung, M. K., Creech-Eakman, M. J., Qi, C., Kessler, J. E., et al. 2001, ApJ, 547, 1077

Chiar, J. E., Adamson, A. J., Pendleton, Y. J., et al. 2002, ApJ, 570, 198

Creech-Eakman, M. J., Chiang, E. I., Joung, R. M. K., Blake, G. A., \& van Dishoeck, E. F. 2002, A\&A, 385, 546

Cutri, R. M., Skrutskie, M. F., van Dyk, S., et al. 2003, 2MASS All Sky Catalog of point sources

Dartois, E., \& d'Hendecourt, L. 2001, A\&A, 365, 144

Dartois, E., d'Hendecourt, L., Thi, W., Pontoppidan, K. M., \& van Dishoeck, E. F. 2002, A\&A, 394, 1057

Davis, S. S. 2007, ApJ, 660, 1580

d'Hendecourt, L. B., Allamandola, L. J., \& Greenberg, J. M. 1985, A\&A, 152, 130

Duley, W. W., \& Williams, D. A. 1984, Nature, 311, 685

Ehrenfreund, P., Gerakines, P. A., Schutte, W. A., van Hemert, M. C., \& van Dishoeck, E. F. 1996, A\&A, 312, 263

Gezari, D. Y., Pitts, P. S., \& Schmitt, M. 1999, 5

Gillett, F. C., \& Forrest, W. J. 1973, ApJ, 179, 483

Gillett, F. C., Jones, T. W., Merrill, K. M., \& Stein, W. A. 1975, A\&A, 45, 77

Graham, J. A. 1992, PASP, 104, 479

Hagen, W., Tielens, A. G. G. M., \& Greenberg, J. M. 1981a, Chem. Phys., 56, 367

Hagen, W., \& Tielens, A. G. G. M. 1981b, Chem. Phys., 75, 4198

Haisch, K. E., Jr., Barsony, M., Greene, T. P., \& Ressler, M. E. 2002, ApJ, 124, 2841

Haisch, K. E., Barsony, M., Ressler, M. E., \& Greene, T. P. 2006, AJ, 132, 2675

Hartquist, T. W., \& Wolf, M. 1990, MNRAS, 247, 343

Jones, T. W., \& Merrill, K. M. 1976, ApJ, 209, 509

Koresko, C. D., Herbst, T. M., \& Leinert, Ch. 1997, ApJ, 480, 741

Kouchi, A., \& Kuroda, T. 1990, Nature, 344, 134 
Léger, A., Jura, M., \& Omont, A. 1985, A\&A, 144, 147

Leggett, S. K., Hawarden, T. G., Currie, M. J., et al. 2003, MNRAS, 345, 144 Leinert, Ch., Beck, T. L., Ligori, S., et al. 2001, A\&A, 369, 215

Lenzen, R., Hartung, M., Brandner, W., et al. 2003, SPIE Proc., 4841, 944

Martin, P. G. 1978, in Cosmic Dust (Clarendon Press)

Merrill, K. M., Russell, R. W., \& Soifer, B. T. 1976, ApJ, 207, 763

Mukai, T. 1986, A\&A, 164, 397

Muñoz Caro, G. M., Meierhenrich, U. J., Schutte, W. A., et al. 2002, Nature, 416, 403

Murakawa, K., Tamura, M., \& Nagata, T. 2000, ApJS, 128, 603

Pickles, A. J. 1998, PASP, 110, 863

Pontoppidan, K., van Dishoeck, E. F., \& Dartois, E. 2004, A\&A, 426, 925

Pontoppidan, K. M., Dullemond, C. P., van Dishoeck, E. F., et al. 2005, ApJ, 622,463

Press, W. H., Teukolsky, S. A., Vetterling, W. T., \& Flannery, B. P. 1986 (UK: Cambridge University Press)

Przygodda, F. 2004, Ph.D. Thesis, Ruprecht-Karls-Universität, Heidelberg Querry, M. R., Curnutte, B., \& Williams, D. 1969, J. Opt. Soc. Am., 59, 1299

Rayner, J. T., Cushing, M. C., \& Vacca, W. D. 2009, ApJS, 185, 289

Rousset, G., Lacombe, F., Puget, P., et al. 2003, SPIE Proc., 4839, 140
Schegerer, A., Wolf, S., Voshchinnikov, N. V., Przygodda, F., \& Kessler-Silacci, J. E. 2006, A\&A, 456, 535

Schmitt, B., Quirico, E., Trotta, F., \& Grundy, W. M. 1998, in Solar System Ices, ed. D. Kluwer (Academic Publishers), 199

Simon, M., Howell, R. R., Longmore, A. J., et al. 1987, ApJ, 320, 344

Smith, R. G., Sellgren, K., \& Tokunaga, A. T. 1988, ApJ, 334, 209

Smith, R. G., Sellgren, K., \& Tokunaga, A. T. 1989, ApJ, 344, 413

Šolc, M. 1980, Acta Math., 21, 19

Stevenson, D. J., \& Lunine, J. I. 1988, Icarus, 75, 146

Teixeira, T. C., \& Emerson, J. P. 1999, A\&A, 351, 292

Terada, H., Tokunaga, A. T., Kobayashi, N., et al. 2007, ApJ, in press

Thi, W.-F., van Dishoeck, E. F., Dartois, E., et al. 2006, A\&A, 449, 251

Tielens, A. G. G. M., \& Hagen, W. 1982, A\&A, 114, 245

Tielens, A. G. G. M., Allamandola, L. J., Bregman, J., et al. 1984, ApJ, 287, 697 van der Bliek, N. S., Manfroid, J., \& Bouchet, P. 1996, A\&AS, 119, 547

van de Bult, C. E. P. M., Mayo Greenberg, J., \& Whittet, D. C. B. 1985, 214, 289

Wallace, L., \& Hinkle, K. 2002, AJ, 124, 3396

Whittet, D. C. B., in Dust In The Galactic Environment 2002, ed. M. Birkinshaw, M. Elvis, \& J. Silk (The Institute of Physics)

Wolf, S., \& Voshchinnikov, N. V. 2004, CoPhC, 162, 113 


\section{Appendix A: Additional measurements}

Table A.1 provides details of additional observations with NAOS-CONICA. The quantity $T_{\text {tot }}$ is the total exposure time. The standard stars HR 6070 and BS 7330 were observed directly before or after the observation of each target. The brightness of the standard star BS 7330 is known from the standard star catalog of Leggett et al. (2003). The observation of the standard star BS 7330 helped to remove the telluric absorption features from the spectrum of VVCrA and its subsequent photometric calibration, while the object HR 6070 was used as a standard star for all remaining objects. The $L$ band brightness of the objects CRBR 2422.8-3423 and VV CrA A are found in the Gezaricatalog (1999). The $L$ band brightness of the infrared companion VV CrA B is not known. The FWHM of the projected diameter of CRBR 2422.8-3423 could be estimated using a $K$ band image (Pontoppidan et al. 2005). The FWHM of the diameter of $\mathrm{VV} \mathrm{CrA} \mathrm{A} \mathrm{and} \mathrm{VV} \mathrm{CrA} \mathrm{B} \mathrm{were} \mathrm{derived} \mathrm{using} \mathrm{interferometric}$ measurements in $N$ band (Przygodda 2004). The flux and the diameter of the object Elia 2-23 in the $L$ band were taken from Haisch et al. (2002). The NICMOS/HST-image of GY92 244 in the $H$ band (Allen et al. 2002) allows us to approximate the diameter of the corresponding disks in $L$ band. The $L$ band brightness of this object was estimated by considering known $H$ band magnitudes and $(H-K)$ and $(K-N)$ colors of additional YSOs in the same star-forming region (Allen et al. 2002).

Analogous to YLW 16 A, all targets were observed twice, with two orthogonal orientations of the slit: parallel (p) and orthogonal (o) to a predefined direction. If the position angle of the disk was not known, the orientation of possible companions were considered in the subsequent selection of the slit orientation. A slit length of $28^{\prime \prime}$ allowed us to observe the companions of VV CrA A (i.e., VV CrA B at an angular distance of 1.69"; Przygodda 2004) and Elia 2-23 (Elia 2-21 at an angular distance of 10"; Haisch et al. 2002) in the parallel orientation of the slit, simultaneously. The data reduction of these observations is described in Sect. 3.

Figures A.1 and A.2 show the resulting $L$ band spectra of these targets. Considering the derived flux, some sources exhibit an intrinsic variability in $L$ band (Leinert et al. 2001). For instance, it is known that the NIR brightness of VV CrA "conspicuously" varies within a period of few years (Graham 1992; Chen \& Graham 1993; Koresko et al. 1997; Przygodda 2004).
The result of our observations of $\mathrm{VV} \mathrm{CrA} \mathrm{A}{ }^{6}$ conflicts with a spectroscopic measurement performed on data acquired using the 4m-telescope of the CTIO (Cerro Tololo Inter-American Observatory; Chen \& Graham 1993). The absorption band of VV CrA A detected by our observation is weak, while the observation of Chen \& Graham (1993) does not show any absorption ice band at all. This difference may be attributed to the factor-oftwo higher spectral resolution power of NAOS-CONICA and its higher spatial resolution power by a factor of $\sim 11$. Therefore, observations with NAOS-CONICA allow us to observe more central regions that are strongly influenced by both the star and disk. The water ice absorption band of the infrared companion is more pronounced. This companion, that is strongly variable in the infrared wavelength range (Koresko et al. 1997), exhibits a broad absorption band. Our analysis of the observations of this source agree with previous measurements with the $4 \mathrm{~m}$-telescope at the CTIO.

Owing to the noisy data from which only parts of the telluric features could be removed, processed water ice, i.e., crystallized and/or grown ice grains cannot unambiguously be found. Residual telluric features also hamper the determination of the column densities $\mathrm{N}_{\mathrm{A}}\left(\mathrm{H}_{2} \mathrm{O}\right.$ [ice]). Figure A.3 shows the optical depths of the objects for which the determination of the column density and a subsequent modelling were possible, given the residual telluric features. Table A.2 lists the corresponding results.

The detection of crystallized water ice in the spectra of CRBR 2422.8-3423 cannot definitely be confirmed. Studying the derived profiles in Smith et al. (1989) and Dartois \& d'Hendecourt (2001), a signal-to-noise ratio greater than $\sim 4$ is required to prove crystallized water ice in the spectra, i.e., to distinguish the extinction profiles of crystallized and amorphous ice grains. Our measurement of CRBR 2422.8-3423 is obtained from data with a signal-to-noise ratio of $\sim 6$. However, the profile of CRBR 2422.8-3423 cannot be used because its absorption is saturated (Fig. A.1).

\footnotetext{
${ }^{6}$ The main component of the binary in $N$ band is the south-west component in the binary system. The north-east component is brighter in $L$ band.
} 



Fig. A.1. Extracted and photometrically calibrated $L$ band spectra of further targets. 
A. A. Schegerer and S. Wolf: Crystallized water ice in T Tauri objects

Table A.1. Overview of additional observations with NAOS-CONICA. Coordinates, $L$ band magnitude, $F W H M$, date, observing time (UT), and the mean airmass $(A M)$ during the observations are listed.

\begin{tabular}{|c|c|c|c|c|c|c|c|c|}
\hline Object & $\alpha(\mathrm{J} 2000)$ & $\delta(\mathrm{J} 2000)$ & $L[\mathrm{mag}]$ & $F W H M["]$ & date & $U T$ & $A M$ & $T_{\text {tot }}$ \\
\hline HR 6070 & 161818 & -283646 & 4.807 & - & April 2nd, 2006 & $7: 02-7: 04$ & 1.0 & $2.0 \mathrm{~s}$ \\
\hline Elia 2-23 (p) & 162624 & -242450 & 5.9 & 2 & Apr. 2nd, 2006 & $7: 45-9: 34$ & 1.0 & $72 \times 6 \times 2.0 \mathrm{~s}$ \\
\hline HR 6070 & 161818 & -283646 & 4.807 & - & April 2nd, 2006 & $7: 02-7: 04$ & 1.0 & $2.0 \mathrm{~s}$ \\
\hline Elia 2-21 & 162624 & -242450 & 8.0 & 1 & April 2nd, 2006 & $7: 45-9: 34$ & 1.0 & $72 \times 6 \times 2.0 \mathrm{~s}$ \\
\hline Elia 2-23 (c & 624 & -242450 & 5.9 & 2 & June 22nd, 2006 & $0: 00-0: 30$ & 1.0 & $76 \times 6 \times 2.0 \mathrm{~s}$ \\
\hline HR 6 & 18 & -283646 & 4.807 & - & June 22nd, 2006 & $0: 45-0: 47$ & 1.0 & $2.0 \mathrm{~s}$ \\
\hline $4(\mathrm{p})$ & 17 & -242858 & 8.8 & 2 & May 27th, 2006 & $2: 48-4: 00$ & 1.1 & $54 \times 18 \times 2.0 \mathrm{~s}$ \\
\hline $\mathrm{HR}$ & 18 & -283 & 4.807 & - & May 27th, 2006 & $4: 24-4: 26$ & 1.0 & $2.0 \mathrm{~s}$ \\
\hline GY92 244 (о) & 17 & -242858 & 8.8 & 2 & May 27th, 2006 & $5: 25-6: 02$ & 1.0 & $36 \times 18 \times 2.0 \mathrm{~s}$ \\
\hline HR 6070 & 161818 & -283646 & 4.807 & - & May 27th, 2006 & $6: 22-6: 24$ & 1.1 & $2.0 \mathrm{~s}$ \\
\hline CRBR 2422.8-3423 (p) & 162705 & -243631 & 9.7 & 1 & August 12th, 2006 & $2: 01-2: 57$ & 1.3 & $18 \times 40 \times 4.0 \mathrm{~s}$ \\
\hline HR 6070 & 161818 & -283646 & 4.807 & - & Aug. 12th, 2006 & $3: 15-3: 17$ & 1.5 & $2.5 \mathrm{~s}$ \\
\hline CRBR 2422. & 162705 & -243631 & 9.7 & 1 & August 19th, 2006 & $0: 14-0: 45$ & 1.0 & $12 \times 60 \times 3.0 \mathrm{~s}$ \\
\hline HR 6070 & 161818 & -283646 & 4.807 & - & August 19th, 2006 & $1: 11-1: 13$ & 1.1 & $2.5 \mathrm{~s}$ \\
\hline VV C & 19 & -37 & 3.7 & 1 & July 27th, 2006 & $5: 23-6: 01$ & 1.2 & $\begin{array}{r}60 \times 8 \times 1.5 \mathrm{~s}+ \\
+16 \times 5 \times 5.0 \mathrm{~s}\end{array}$ \\
\hline BS 7 & & -34 & 4.9 & - & 2006 & 30 & 1.3 & $2.0 \mathrm{~s}$ \\
\hline VV CrA A (o) & & -371 & 3.7 & 1 & July 4th, 2006 & $5: 17-6: 03$ & 1.0 & $60 \times 12 \times 1.0 \mathrm{~s}$ \\
\hline BS 7330 & 192129 & -345857 & 4.9 & - & July 4th, 2006 & $6: 20-6: 22$ & 1.1 & $2.0 \mathrm{~s}$ \\
\hline VV CrA B & 190307 & -371250 & 6.2 & 1 & July 27th, 2006 & $5: 23-6: 01$ & 1.2 & $\begin{array}{l}60 \times 8 \times 1.5 \mathrm{~s}+ \\
+16 \times 5 \times 5.0 \mathrm{~s}\end{array}$ \\
\hline BS 7330 & 192129 & -345857 & 4.9 & - & July $27^{\text {th }}, 2006$ & $6: 28-6: 30$ & 1.3 & $2.0 \mathrm{~s}$ \\
\hline
\end{tabular}

Table A.2. Column density $N_{\mathrm{A}}$ of water ice along the line of sight to the observer (Eq. (4)).

\begin{tabular}{lccccccc}
\hline \hline Exposure & $P A\left[{ }^{\circ}\right]$ & $N_{\mathrm{A}}\left[10^{22} \mathrm{~m}^{-2}\right]$ & $A_{\mathrm{V}}[\mathrm{mag}]$ & $m_{\mathrm{a} ; \mathrm{s}}[\%]$ & $m_{\mathrm{a} ; 1}[\%]$ & $m_{\mathrm{c}}[\%]$ & $\chi$ \\
\hline Elia 2-23 (p) & 53 & 0.52 & 7 & - & - & - & - \\
Elia 2-23 (o) & 45 & 0.26 & 5 & 100 & - & - & 0.04 \\
Elia 2-21 (p) & 53 & 0.66 & 9 & - & - & - & - \\
GY92 244 (p) & 178 & 1.8 & 20 & $>99$ & $<1$ & $<1$ & 0.08 \\
GY92 244 (o) & 88 & 2.1 & 23 & $>99$ & - & $<1$ & 0.1 \\
CRBR 2422.8-3423 (p) & 53 & 3 & 35.1 & - & - & - & - \\
CRBR 2422.8-3423 (o) & 45 & 3 & 29.1 & 91 & $<1$ & 8 & 0.2 \\
VV CrA B (p) & 134 & 0.66 & 9 & $>99$ & - & $<1$ & 0.05 \\
VV CrA A (p) & 134 & 0.13 & 3 & - & - & - & - \\
VV CrA A (o) & 43 & 0.030 & 2 & - & - & - & - \\
\hline
\end{tabular}


A\&A 517, A87 (2010)


Fig. A.2. Continuation of Fig. A.1. 



Fig. A.3. Optical depth $\tau(\lambda)$ that is derived from the spectra of Fig. A.1 and Fig. A.2. The optical depth of the spectra Elias 2-23 (p), Elias 2-23 (o), Elias 2-21, CRBR 2422.8-2423 (p), VV CrA (p), and VV CrA (o) are not modeled because of low signal-to-noise ratios and remaining telluric features that could not be sufficiently eliminated in data reduction. 